\title{
Laser diffractometry of nanoparticles: frequent pitfalls \& overlooked opportunities
}

\author{
Senem Acar Kübart ${ }^{1}$ and Cornelia M. Keck ${ }^{1,2^{*}}$ \\ *Correspondence: cornelia.keck@fh-kl.de \\ 'Department of Pharmaceutics, Biopharmaceutics \& NutriCosmetics, Freie Universität Berlin, Kelchstr. 31, 12169 Berlin, \\ Germany. \\ ${ }^{2}$ Applied Pharmacy Division, Department of Applied Logistics and Polymer Sciences, University of Applied Sciences \\ Kaiserslautern, Carl-Schurz-Str. 10-16, 66953 Pirmasens, Germany.
}

\begin{abstract}
Laser diffraction is a frequently applied technique for the size analysis of particles. The method possesses many advantages but also disadvantages or pitfalls. If these pitfalls are overlooked or not considered appropriately, size analysis by laser diffraction can lead to false and/or meaningless results. As shown in previous studies, this is especially true for the size analysis of nanoparticles. In this study further possible pitfalls for the size analysis of nanosized formulations were investigated. This included both, influences related to the sampling and influences related to the instrument setup. The results revealed that sampling position, the type of sampling device, the stirring speed in the instrument and/or the use of ultrasound can lead to tremendous changes in the size result. However, the data also showed that these often overlooked pitfalls, if understood, represent a great opportunity to gain more detailed information about the properties of the nanosized formulations.
\end{abstract}

Keywords: Lipid nanoparticles, laser diffractometrym, particle size, sampling position, sampling device, instrumental setup, stirring speed, ultrasound

\section{Background}

The use of nanocarriers has become an important delivery principle in pharmaceutics and many other fields. Examples of pharmaceutical nanocarriers are nanoemulsions [1-3], liposomes [4,5], polymeric nanoparticles [6-8], drug nanocrystals [9], or lipid nanoparticles [10]. Due to their small size, nanoparticles in comparison to micro- or macroparticles, possess special properties, which can be exploited for drug delivery. Examples are an increased dissolution rate and solubility as observed for drug nanocrystals [11], increased oral bioavailability in case of lipidic nanocarriers [10] or less undesired side effects [12]. Obviously, all these effects depend on the size of these particles. Therefore, the most important pre-requisite for a successful development of effective nanocarriers is, to obtain an appropriate size and to prevent changes over time, i.e., to ensure physical stability during the shelf life of the product. For this the particle size needs to be correctly analyzed.

Today many different sizing methods are employed for the characterization of nanoparticles. Examples are microscopic methods (eg. scanning electron microscopy, transmission electron microscopy or atomic force microscopy), centrifugal sedimentation, field flow fractionation or light scattering techniques (eg. dynamic or static light scattering). Each of these methods has advantages but also disadvantages and none of these techniques is capable to fully characterize a nanosystem. An example is dynamic light scattering (DLS), also known as photon correlation spectroscopy (PCS). The technique is advantageous because it can analyze nanoparticles very accurately and highly reproducible. The method is inexpensive and fast. Therefore today, this technique is the most frequently technique used for size analysis of nanoparticles. However, it can analyze only particles below $6 \mu \mathrm{m}$ [13]. Hence, possible larger particles, eg. agglomerates cannot be detected using this method. Therefore, other techniques must be used for the detection of possible larger particles. The ability to detect possible larger particles within a nanosystem is extremely important, because larger particles are unwanted in a nanosystem and change the (nano-) properties of the formulation or product. Techniques for the detection of larger particles within a nanosized system are eg. light microscopy or static light scattering techniques. The disadvantage of light microscopy is the need to analyze a sufficient high number of particles to obtain a significant result. Therefore, in praxis very often static light scattering, eg. laser diffraction is used for the characterization of nanoparticles containing possibly larger particles.

Laser diffractometry (LD) has many advantages, eg. it is a fast and inexpensive analysis method, it possesses a broad measuring range (eg. $20 \mathrm{~nm}-2000 \mu \mathrm{m})$ and can therefore analyze nanoparticles, microparticles and/or mixtures of them. By using laser diffraction solid and liquid samples can be analyzed. Hence, almost all types of samples can be analyzed by this technique. Nevertheless, also this 


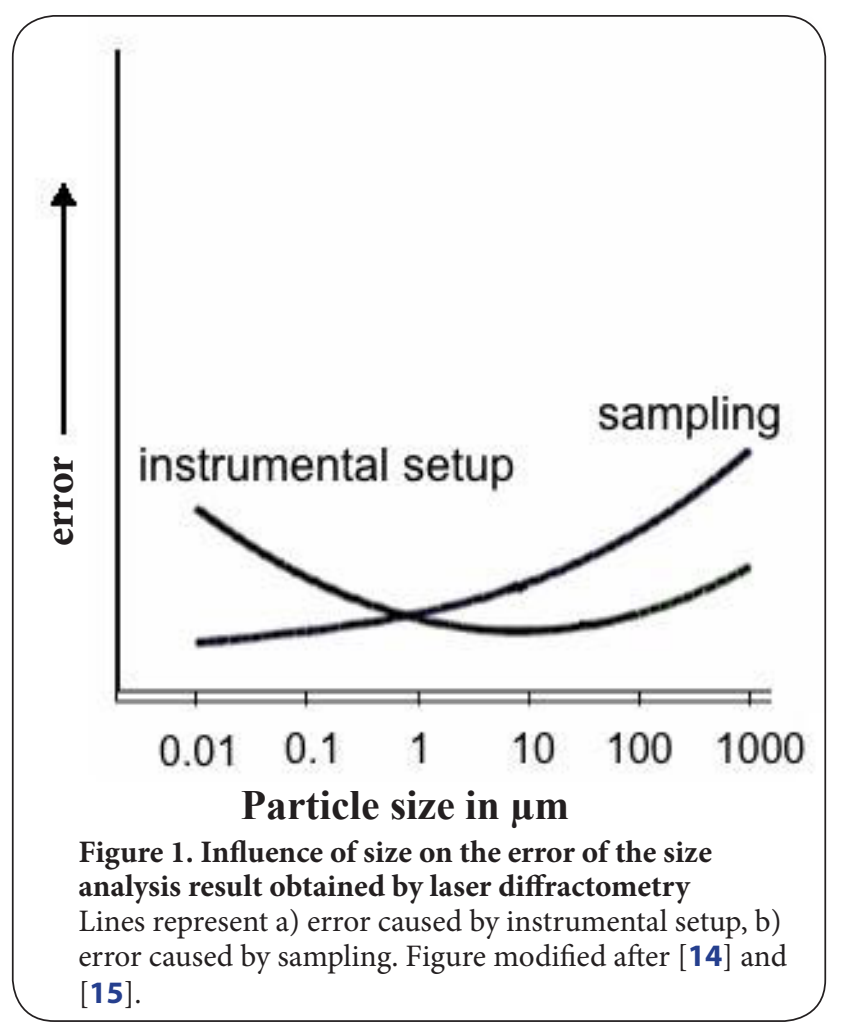

technique has disadvantages. In principle all these pitfalls or disadvantages can be divided into problems related to the sampling or into problems related to the instrumental setup. After T. Allen [14] pitfalls and errors related to the sampling increase with increasing sample size, whereas pitfalls related to the technique or the instrumental setup decrease with increasing size are less (Figure 1).

Based on this theory it can be assumed that samples containing nanoparticles and larger particles at the same time, lead to large errors for both, the instrumental error and the error due to sampling. However, when working with nanocarriers these kinds of samples are frequently analyzed, eg. during formulation development or during storage. A meaningful analysis of such samples is thus very important, as it is a prerequisite to discriminate samples from each other. For example, if size analysis can reliably detect differences in the mean size and/or the number larger particles or agglomerates, reasons causing these differences, eg. production parameters, concentration and type of stabilizers, storage conditions, etc. can be identified and optimized. Cleary, an early discrimination between "good and bad" samples improves the formulations development by saving time and costs. However, reproducible and meaningful size results can only be obtained if the pitfalls of a sizing method are understood and if ways of how to overcome these pitfalls are known.

Possible pitfalls for the size analysis of nanoparticles using laser diffractometry were already investigated in previous studies.
From these studies the following important pitfalls were identified:

- The use of the Fraunhofer approximation or the Mietheory with incorrect optical parameters for the size analysis [16].

- The overestimation of small sized particles in a system containing nanoparticles and larger particles at the same time due to additional techniques used to extend the measuring range to the nanometer range [16].

- Instability of the sample during the measurement (eg. dissolution of drug nanocrystals) [17].

These pitfalls, if not considered and dealt with correctly, can lead to false and/or non-reproducible results. Methods how to detect and how to overcome them are discussed in [16-18].

The aim of this study was to investigate further possible pitfalls during size analysis of nanoparticles by laser diffractometry. To identify such possible pitfalls the measurement procedure was analyzed critically and procedures during the measurement which were often subject to change (eg. by different operators) were monitored closely.

As a result from these observations the following critical points were identified:

- The way how to draw the sample: samples were collected from different positions (bottom, middle or top).

- Type of sampling device: different types of pipettes were used.

- Stirring speed during the measurement: different stirring speeds with and without ultrasound were applied.

These parameters and their possible influences on the size results were studied systematically.

\section{Materials and Methods \\ Materials \\ Samples}

Different nanostructured lipid nanoparticles (NLC) were used as samples in this study. Samples were arranged in three groups, i.e., group A, B and C (cf. Section 2.2.2). (Table 1) provides a list of all samples investigated and there compositions.

The solid lipids Softisan ${ }^{\circledR} 154$ (hydrogenated palm oil) and Dynasan ${ }^{\circledast} 118$ (microcrystalline tristearin) were a kind gift from Sasol GmbH, Germany. Cutina ${ }^{\circledR} \mathrm{CT}$ (cetyl palmitate) was obtained from Cognis Deutschland $\mathrm{GmbH}$, Germany. Miglyol $^{\circledR} 812$ (medium chain triglycerides) as liquid lipid and menthol as model drug were obtained from Caesar \& Loretz $\mathrm{GmbH}$, Germany. As stabilizer either Inutec ${ }^{\circledR}$ SP1 (inulin lauryl carbamate; Orafti Bio Based Chemicals, Belgium), PlantaCare 2000 UP (decyl glucoside; Cognis GmbH, Germany), Pluronic ${ }^{\circledR}$ F68 (polyethylene-polypropylene glycol; BASF, Germany), TegoCare ${ }^{\circledR} 450$ (polyglyceryl-3 methylglucose distearate; Goldschmidt GmbH, Germany) or Tween ${ }^{\circledast} 80$ (polyoxyethylene sorbitan monooleate; 
Kubart et al. Journal of Pharmaceutical Technology \& Drug Research 2013, http://www.hoajonline.com/journals/pdf/2050-120X-2-17.pdf

Table 1. Sample investigated, sample codes and compositions. Composition is given in $\%(w / w)$, samples were made up to $100.0 \%$ with water.

\begin{tabular}{|c|c|c|c|c|c|c|c|}
\hline \multirow[t]{3}{*}{ group } & \multirow{3}{*}{$\begin{array}{c}\text { sample } \\
\mathrm{A} 1\end{array}$} & \multicolumn{6}{|c|}{ composition $(\%-w / w)$} \\
\hline & & \multicolumn{2}{|c|}{ solid lipid } & \multirow{2}{*}{$\begin{array}{c}\text { Miglyol } \mathbf{8 1 2} \\
8.0 \%\end{array}$} & \multirow{2}{*}{$\frac{\text { menthol }}{4.0 \%}$} & \multicolumn{2}{|c|}{ stabilizer } \\
\hline & & Softisan 154 & $10.0 \%$ & & & TegoCare ${ }^{\star} 450$ & $1.8 \%$ \\
\hline \multirow{7}{*}{ A } & $\mathrm{A} 2$ & Dynasan $^{\oplus} 118$ & $7.0 \%$ & $7.0 \%$ & $6.0 \%$ & TegoCare ${ }^{\star} 450$ & $1.8 \%$ \\
\hline & A3 & Dynasan $^{\varpi} 118$ & $8.0 \%$ & $6.0 \%$ & $6.0 \%$ & TegoCare ${ }^{\circledast} 450$ & $1.8 \%$ \\
\hline & A5 & Softisan ${ }^{\circledR} 154$ & $10.2 \%$ & $6.8 \%$ & $3.0 \%$ & TegoCare ${ }^{\star} 450$ & $1.0 \%$ \\
\hline & A6 & Softisan ${ }^{\triangleright} 154$ & $10.2 \%$ & $6.8 \%$ & $3.0 \%$ & Tween 80 & $1.0 \%$ \\
\hline & A7 & Softisan ${ }^{\varpi} 154$ & $10.0 \%$ & $8.0 \%$ & $2.0 \%$ & PlantaCare ${ }^{\circledR} 2000$ UP & $1.8 \%$ \\
\hline & A9 & Dynasan $^{\circledast} 118$ & $9.0 \%$ & $9.0 \%$ & $2.0 \%$ & "PlantaCare ${ }^{\circledR} 2000$ UP & $1.8 \%$ \\
\hline & $\mathrm{A} 10$ & Softisan 154 & $7.0 \%$ & $0.0 \%$ & $3.0 \%$ & TegoCare ${ }^{\circledast} 450$ & $1.8 \%$ \\
\hline \multirow{5}{*}{ B } & B1 & Softisan 154 & $10.0 \%$ & $8.0 \%$ & $2.0 \%$ & TegoCare ${ }^{\varpi} 450$ & $1.8 \%$ \\
\hline & $\mathrm{B} 2$ & Dynasan $^{\oplus} 18$ & $10.0 \%$ & $8.0 \%$ & $2.0 \%$ & TegoCare ${ }^{\star} 450$ & $1.8 \%$ \\
\hline & B3 & Dynasan $^{\oplus} 118$ & $9.0 \%$ & $9.0 \%$ & $2.0 \%$ & TegoCare ${ }^{\circledast} 450$ & $1.8 \%$ \\
\hline & B4 & Dynasan 118 & $8.0 \%$ & $8.0 \%$ & $4.0 \%$ & TegoCare ${ }^{\varpi} 450$ & $1.8 \%$ \\
\hline & B5 & Softisan ${ }^{*} 154$ & $8.0 \%$ & $0.0 \%$ & $2.0 \%$ & TegoCare ${ }^{\star} 450$ & $1.8 \%$ \\
\hline \multirow{4}{*}{$\mathrm{C}$} & $\mathrm{Cl}$ & Softisan ${ }^{\circledast} 154$ & $10.2 \%$ & $6.8 \%$ & $3.0 \%$ & Pluronic ${ }^{\circledR} \mathrm{F} 68$ & $1.0 \%$ \\
\hline & $\mathrm{C} 2$ & Softisan ${ }^{\varpi} 154$ & $10.2 \%$ & $6.8 \%$ & $3.0 \%$ & Inutec ${ }^{\circledR} \mathrm{SP} 1$ & $1.0 \%$ \\
\hline & $\mathrm{C} 3$ & Softisan ${ }^{\circledast} 154$ & $10.0 \%$ & $0.0 \%$ & $0.0 \%$ & TegoCare ${ }^{\varpi} 450$ & $1.8 \%$ \\
\hline & $\mathrm{C} 4$ & Softisan ${ }^{\circledR} 154$ & $15.0 \%$ & $5.0 \%$ & $0.0 \%$ & Tween ${ }^{\circledR} 80$ & $1.8 \%$ \\
\hline
\end{tabular}

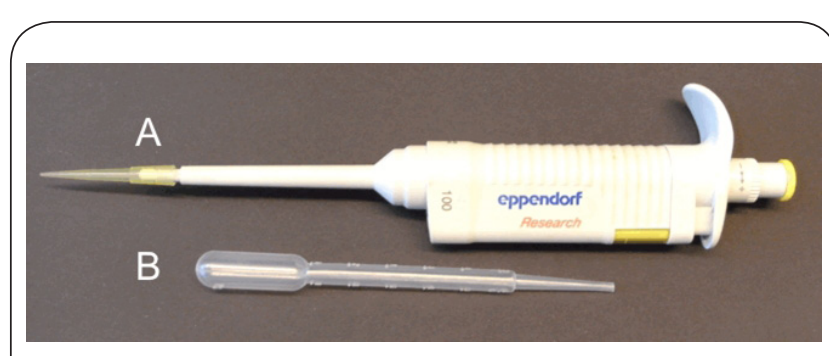

Figure 2. Sampling devices used A: Eppendorfpipette, B: Pasteur pipette

Uniqema, Belgium) was used. For all productions purified water (Milli-Q, Millipore GmbH, Germany) was used.

\section{Sampling devices}

As sampling devices Eppendorf pipettes (model: Research ${ }^{\oplus}$, 10-100 $\mu \mathrm{l}$; Eppendorf AG, Germany) with yellow universal pipette tips (mouth diameter: $0.52 \mathrm{~mm}$; VWR International $\mathrm{GmbH}$, Germany, Figure 2A) and Pasteur pipettes (mouth diameter: about $1.50 \mathrm{~mm}$, made from low-density polyethylene (LDPE), model: High Performance, general purpose; VWR International GmbH, Germany, Figure 2B) were used.

\section{Methods}

\section{Production of nanoparticles}

Nanoparticles were produced by hot high pressure homogenization using an LAB 40 (APV Deutschland GmbH) in discontinuous mode. The production conditions applied were 2 homogenization cycles, 800 bar homogenization pressure and $80^{\circ} \mathrm{C}$ production temperature. Samples were stored in glass vials (silanized glas type II) at room temperature until they were used.
Table 2. Overview of sample groups investigated and experiments performed.

\begin{tabular}{|c|c|c|c|c|}
\hline \multirow[b]{2}{*}{ group } & \multirow[b]{2}{*}{ type of sample } & \multicolumn{3}{|c|}{ experiments performed: } \\
\hline & & $\begin{array}{l}\text { part 1: } \\
\text { influence of } \\
\text { sampling } \\
\text { position }\end{array}$ & $\begin{array}{l}\text { part 2: } \\
\text { influence } \\
\text { of sampling } \\
\text { instrument }\end{array}$ & $\begin{array}{l}\text { part 3: } \\
\text { influence of } \\
\text { stirring force }\end{array}$ \\
\hline $\mathbf{A}$ & $\begin{array}{l}\text { - no } \mu \mathrm{m} \text { particles } \\
\text { - no agglomerates }\end{array}$ & - & sample: 10 & samples: A1-10 \\
\hline B & $\begin{array}{l}\text { - no } \mu \mathrm{m} \text { particles } \\
\text { - slight agglomeration }\end{array}$ & - & sample: B5 & samples: B1-B5 \\
\hline C & $\begin{array}{l}\text { - some } \mu \mathrm{m} \text { particles } \\
\text { and/or } \\
\text { - heavy agglomeration }\end{array}$ & sample: $\mathrm{C} 4$ & sample: C3 & samples: C1-C3 \\
\hline
\end{tabular}

\section{Selection of samples}

The aim of this study was to investigate the influence of sampling position, sampling device and stirring speed or agitation power on the particle size result obtained. To enable the detection of possible effects eg. detection of agglomerates, de-agglomeration or agglomeration of particles during the measurements, it was necessary to select different types of samples. The three groups of samples consisted of small sized non-agglomerated samples (group A), slightly agglomerated samples (group B) and samples containing heavily agglomerated particles (group C). The selection aimed at simulating all possible types of samples which can occur during the development or production of nanocarriers. Possible effects eg. dissolution of the particles during the measurement were not investigated in this study, as it has been studied earlier $[17,18]$. Therefore, only non-dissolving lipid nanoparticles were selected (Table 2). The selection of the samples was performed using light microscopy. Samples containing no visible larger particles or agglomerates at a $160 x$ fold magnification were selected into group $A$, samples containing slight or some 
loose agglomerates into group $B$ and samples with larger or heavily agglomerated particles into group C (cf. Table 2).

\section{Characterization}

Light microscopy

Miroscopic analysis was performed using an Leitz Ortophlan (Leitz, Germany) with different magnifications (160x, 400x, 630x and 1,000x). Images were taken using a CMEX3200 digital camera (Euromex Microscopes, Netherlands) system.

\section{Dynamic light scattering}

Dynamic light scattering (DLS), also known as photon correlation spectroscopy, was performed using a Zetasizer Nano ZS (Malvern Instruments, UK). The samples were measured by applying 10 subsequent measurements with a measurement time of $15 \mathrm{~s}$ each. The size results were calculated using the general purpose mode. The results presented represent the average of the 10 single measurements.

\section{Laser diffractometry}

Laser diffractometry (LD) was performed using a Mastersizer 2000 (Malvern Instruments, UK). For the first part of the study (influence of sampling position) a LS 230 (Beckman Coulter, Germany) was used. All measurements were performed with the additional technique (eg. with polarization intensity differential scattering (PIDS) in case of the LS 230 and blue-light detection system in case of the Mastersizer). The size analysis was performed using the Mie theory with the optical parameters 1.456 for the real refractive index and 0.001 for the imaginary index. As size parameters the median diameter $50 \%(\mathrm{~d}(\mathrm{v}) 0.50)$ and the median diameter $95 \%(\mathrm{~d}(\mathrm{v}) 0.95)$ are presented. The $d(v) 0.50$ represents the size at which $50 \%$ of the volume of the particles are below the given number. It therefore indicates the average of the particle size of the sample. The $d(v) 0.95$ consequently represents the size at which $95 \%$ of the sample are below the given number. Hence, only $5 \%$ of the volume of the particles is above this value. Therefore, the $d(v) 0.95$ is a sensitive measure for possible larger particles in the sample. Measuring conditions (i.e., sampling position, type of sampling device, stirring rate and ultrasound during the measurement) were varied for each part of the study. A detailed description of the measuring conditions applied is described below.

\section{Part 1: Influence of sampling position}

In this part of the study the effect of the sampling position was varied (upper, lower, middle (all non-shaken), and shaken), whereas all other conditions were kept constant. Samples were drawn from the original vial containing about $30 \mathrm{ml}$ of sample C4. Prior to the sampling the vial was left without any motion for $24 \mathrm{~h}$, to allow the particles to float or sediment. For each sampling position a sample amount of about $1 \mathrm{ml}$ was collected using a syringe $(2 \mathrm{ml})$ with a needle $(60 \times 1 \mathrm{~mm})$. First the sample from the upper position was carefully collected from the surface of the dispersion. The sample representing the middle of the sample was than collected from the centre of the vial and the third sample was obtained from the bottom of the vial, representing the "lower" sampling position. Between the samplings the dispersion was rested for $30 \mathrm{~min}$ in order to minimise the disturbance of particles due to the previous sampling. Finally, the sample was gently shaken for $30 \mathrm{~s}$ by hand and the sample "shaken" was collected from the center of the vial. The differently collected samples of sample C4 were than analyzed using the LS 230 (stirring speed $50 \mathrm{rpm}$ ). In this instrument the maximum stirring speed is $100 \mathrm{rpm}$. However, typically $50 \mathrm{rpm}$ are used, sonication is not possible in the instrument. The sample was added using an Eppendorf pipette. Each measurement was performed in triplicate and each measurement consisted of three subsequent runs.

\section{Part 2: Influence of sampling instrument}

In this part the samples (A10, B4, C3) were measured using the Mastersizer 2000 with its standard measuring conditions, i.e., with blue light detection system included, stirring rate $2975 \mathrm{rpm}$, no sonication, four subsequent measurements. Samples were gently shaken by hand for $30 \mathrm{~s}$ before sampling. The samples were added with either an Eppendorf pipette or a Pasteur pipette (Figure 2).

\section{Part 3: Influence of stirring and ultrasound}

Samples (A1-A9, B1-B4, C1 and C2) were shaken by hand for about $30 \mathrm{~s}$ and were added to the instrument using an Eppendorf pipette.

The stirring speed and agitation during the measurement was varied as follows:

- Method 1 (M1): stirring speed 1085 rpm, no sonication.

- Method 2 (M2): stirring speed 2975 rpm, no sonication.

- Method 3 (M3): stirring speed 2975 rpm with sonication. If not stated differently, the results represent the average of 4 subsequent measurements of one sample drawn.

\section{Results and Discussion}

\section{Influence of sampling position}

The results of the first part of the study are shown in Figure 3. Figure 3-left shows the microscopic image of the sample. It contains some diffuse agglomerates and small sized nanoparticles, which was confirmed by DLS (z-average: $247 \mathrm{~nm}$ ). Figure 3-right shows the results obtained by laser diffractometry. Cleary, the way how is the sample collected from the storage container (eg. the vial) can tremendously influence the size results obtained. The most pronounced differences were found for $\mathrm{d}(\mathrm{v}) 0.95$ values, which represent the amount of larger particles within the sample. The largest values (about $40 \mu \mathrm{m}$ ) were found when the sample was collected from the bottom of the vial (lower position). The $\mathrm{d}(\mathrm{v}) 0.95$ decreased with increasing sampling position. 

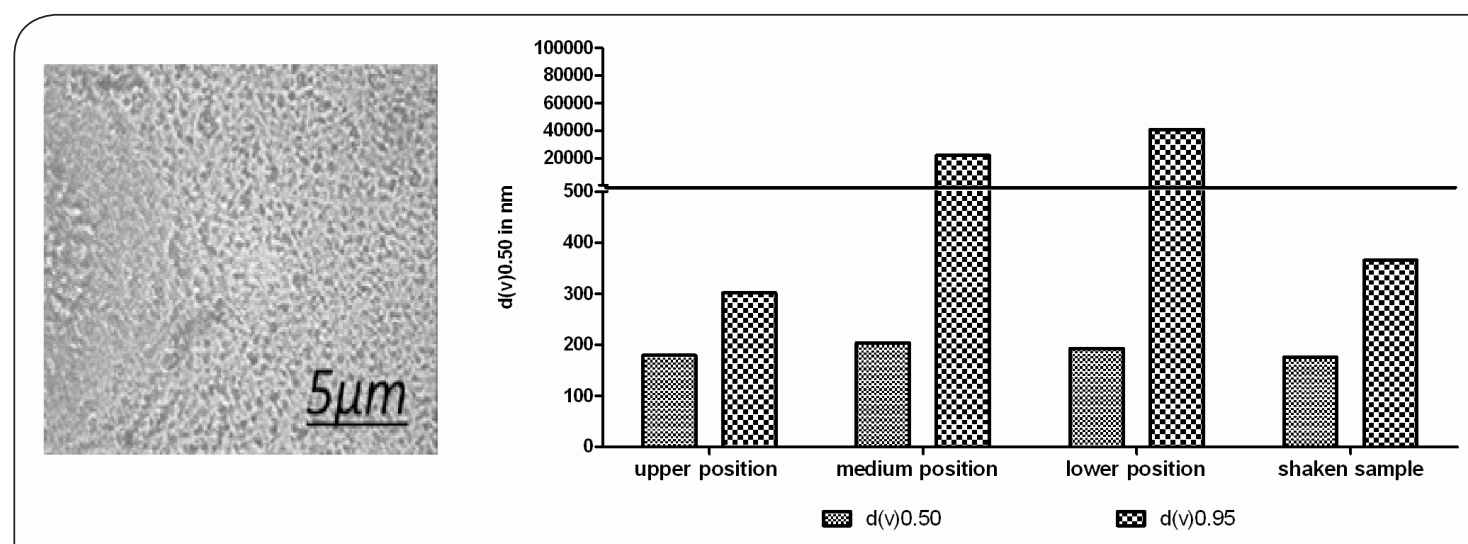

Figure 3. Microscopic image and size results obtained by laser diffractometry of sample C4. Left: microscopic image (magnification 1000x); right: size results obtained by laser diffractometry using different sampling positions, explanation cf. text.

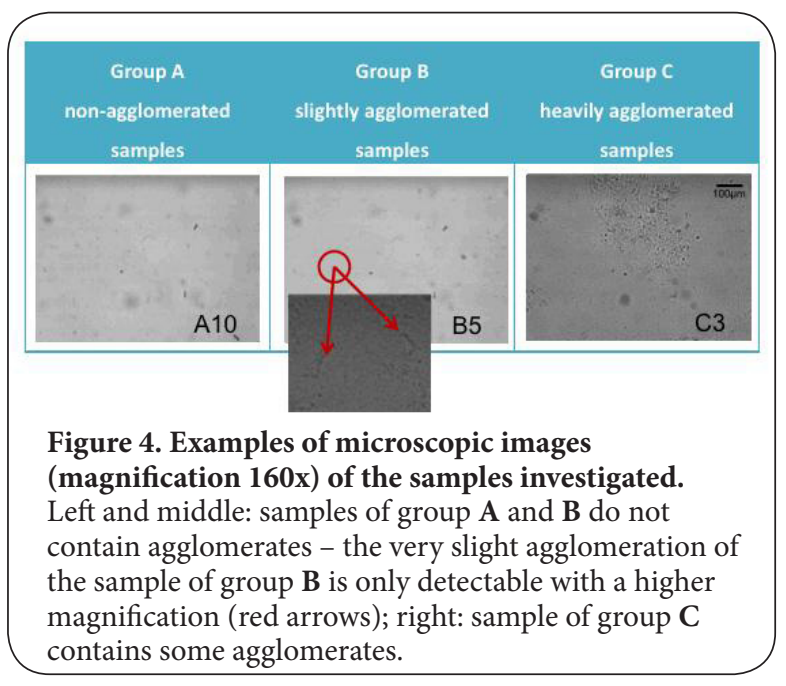

It was about $22 \mu \mathrm{m}$ when the sample was collected from the middle of the vial and about $300 \mathrm{~nm}$ when the sample was collected from the top of the vial. A similar trend was obtained for the $\mathrm{d}(\mathrm{v}) 0.50$ values, however to a much less pronounced influence as observed for the $\mathrm{d}(\mathrm{v}) 0.95$ values. The results can be explained by the inhomogeneity of the sample. Larger particles sediment, thus the size of the sample collected is largest. This is because this fraction contains more larger particles than samples collected from the middle or the top of the vial. The particle size obtained from the shaken sample is larger than the size obtained from the "upper position" sample und it is much smaller than the samples collected from the lower and middle position, which is especially true for the $d(v) 0.95$ values. The result supports that larger particles, i.e., agglomerates might be destroyed during the shaking of the sample. Therefore, it is not simply the average size of the results obtained from the lower, middle and upper position (Figure 3-right).

The results prove that the way how the sample is treated prior to the sampling (shaken, non-shaken), as well as the sampling position are crucial pitfalls in size analysis by laser diffractometry. Sampling from different positions can lead to different size results and thus to non-reproducible measurements. However, it also opens the opportunity to gain important information about the properties of a sample, which might not be detectable by other conventionally used techniques. Inhomogeneous samples lead to different results when samples are drawn from different position. For example, if a suspension is physically unstable and tends to form larger particles over time, these larger particles would sediment (or float). Therefore, by analyzing samples from lower (upper) positions which represent an enriched fraction of larger particles, it could be possible to detect even a few larger particles, which would be below the detection limit in case a shaken sample would be analyzed instead. This means a possible instability of a sample could be detected at an earlier state of the development. Therefore in a stability study, analysis of non-shaken samples is an opportunity to get early insight in instabilities.

\section{Influence of sampling device}

\section{Analysis by light microscopy and dynamic light} scattering

Figure 4 shows the microscopic images of the samples analyzed in this part of the study. The first sample (A10) was a non-agglomerated sample containing no larger particles. The second sample (B5) was also non-agglomerated when analyzed at a $160 x$ fold magnification. However, by enlarging the image, a very slight agglomeration was detected (Figure 4-middle - red arrows). The third sample (C3) clearly contained a large agglomerate with a size $>200 \mu \mathrm{m}$. DLS data (Figure 5) obtained showed no difference in size between the first and the second sample (A10 and B5, respectively). For the third sample (C3) a slightly larger DLS size of about $300 \mathrm{~nm}$ was obtained. However, no indication about the agglomerates found in C3 from light microscopy, are detected by this sizing method (upper detection limit $6 \mu \mathrm{m})$. 


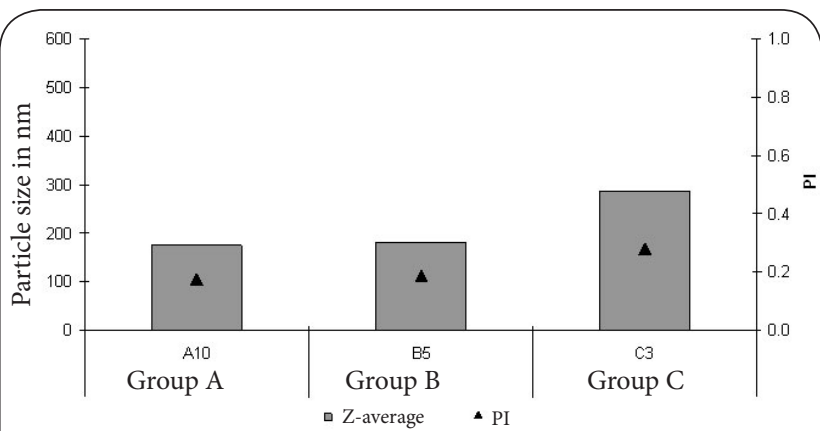

Figure 5. Particle size (z-average) and size distribution (polydispersity index) assessed by dynamic light scattering.

No significant difference in size and size distribution is obtained for the samples of group $\mathbf{A}$ and $\mathbf{B}$, the sample of group $\mathbf{C}$ is larger.

\section{Size analysis by laser diffractometry}

Figure 6 shows the results obtained when the samples were analyzed by laser diffractometry using different sampling instruments (Eppendorf pipette vs. Pasteur pipette, cf. Figure 2) and standard measuring conditions (stirring speed 2975 rpm, no ultrasound). Figure 6-left shows the $d(v) 0.50$ values and Figure 6-right shows the $d(v) 0.95$ values. No differences in the size results were obtained for the non-agglomerated sample A10. Slightly higher values (for both diameters $d(v) 0.50$ and $d(v) 0.95$ ) were obtained for the very slightly aggregated sample B5, when the Eppendorf pipette was used instead of the Pasteur pipette. This effect was even more pronounced (especially true for the $\mathrm{d}(\mathrm{v}) 0.50$ ) for sample $\mathrm{C} 3$, containing larger aggregates, as seen from light microscopy.

The differences in the results are probably related to the different diameters and/or shapes of the tips. The diameter of the tip of the Eppendorf pipette is $0.52 \mathrm{~mm}$ (manufacturer information) with a tapered shape, whereas the mouth diameter of the Pasteur pipette is about $1.5 \mathrm{~mm}$ and a less tapered shape. Due to this, during addition of the particles to the instrument, the forces (eg. shearing or squeezing) acting on the particles are probably slightly higher in case an Eppendorf pipette is used instead of a Pasteur pipette. At the first glance it was expected, that these higher forces would lead to at least some de-aggregation of loose agglomerates, eg. observed for the slightly agglomerated sample B5, whereas it was expected that these forces are not sufficient to destroy tighter agglomerates, eg. the agglomerates seen for sample C3. In this case smaller size results would have been obtained for sample B5 when added to the instrument with an Eppendorf pipette and larger size results when added with the Pasteur pipette respectively. For the agglomerated sample C3 similar results for both sampling instruments were expected, or if the agglomerates were loose, similar to sample B5, smaller sizes for the addition with the Eppendorf pipette were expected. However, these results were not obtained. As the particle size obtained using the Eppendorf pipette with higher forces is higher, the use of Eppendorf pipettes as sampling instrument tends to force agglomeration of particles. This observation was unexpected, but can be explained by the DLVO theory. During the addition of the particles, especially at the point where they get squeezed out of the pipette, they become accelerated and can get into close contact to each other. Based on the DLVO theory $[19,20]$, the accelerating energy might be sufficient to overcome the critical distance between the particles where the repulsing forces between the particles become less and at the same time the attracting forces (eg. van der Waals forces) become dominant. Consequently, this would lead to the agglomeration of the particles [21]. The effect would be observed in case the stabilizer of the particles is not capable to provide sufficient electrostatic or steric stabilization.

To verify this effect the particles were analyzed again using the two different sampling instruments and two different measuring conditions. The standard measuring method is performed using a stirrer speed of $2975 \mathrm{rpm}$. Stirring is typically applied to constantly circulate the particles through the measuring cell. This is important to ensure a constant number of particles throughout the entire measurement. Otherwise larger particle would sediment (or float) in the measuring cell during the measurement, leading to false results. However, in theory in case loose agglomerates are present within the sample, these forces might also lead to de-agglomeration of the sample. The Mastersizer 2000, used in this study (and most of the modern LD instruments), enables the selection of the stirrer speed. Therefore, to investigate if agglomerates are formed during the addition of the sample when using an Eppendorf pipette, the samples were analyzed using a low stirring rate (1085 rpm = measurement method 1 (M1)), leading to less forces and thus probably to less de-aggregation in case agglomerates would have formed during the addition. The results obtained where compared to the results obtained when applying standard measuring conditions, i.e., 2975 rpm (= measuring method $2(\mathrm{M} 2)$ ). The results are shown in (Figure 7).

For sample A10 (non-agglomerated sample), no difference was found between $M 1$ and $M 2$ for the $d(v) 0.50$ (Figure 7, left). However, the $\mathrm{d}(\mathrm{v}) 0.95$ (Figure 7, right) was about $20 \mu \mathrm{m}$ for M1 and only $239 \mathrm{~nm}$ for M2. Because no such large particles or agglomerates were detected by light microscopy (cf. Figure 4; microscope) and no such effect was observed when the Pasteur pipette was used as sampling instrument, the results nicely confirm the results from above. The effect could be further confirmed by the results obtained for sample B5 (very slight agglomeration). Here, not only the $\mathrm{d}(\mathrm{v}) 0.95$ was affected, but also the $\mathrm{d}(\mathrm{v}) 0.50$. Hence, the degree of agglomeration indicates the sensitivity to of the sample to form agglomerates, 

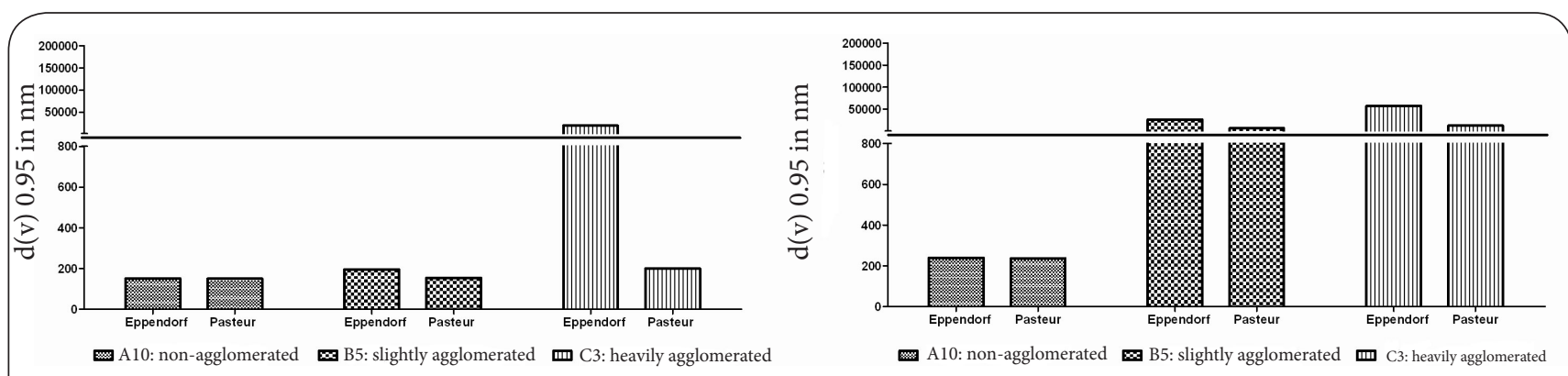

Figure 6. Particle size analysis by laser diffractometry.

Plotted are the $\mathrm{d}(\mathrm{v}) 0.50$ (left), and the $\mathrm{d}(\mathrm{v}) 0.95$ (right). Samples were added to the instrument (Mastersizer) by different sampling devices (Eppendorf pipette or Pasteur pipette, respectively) and analyzed using the standard measuring conditions (no ultrasound, stirring rate $2975 \mathrm{rpm})$.
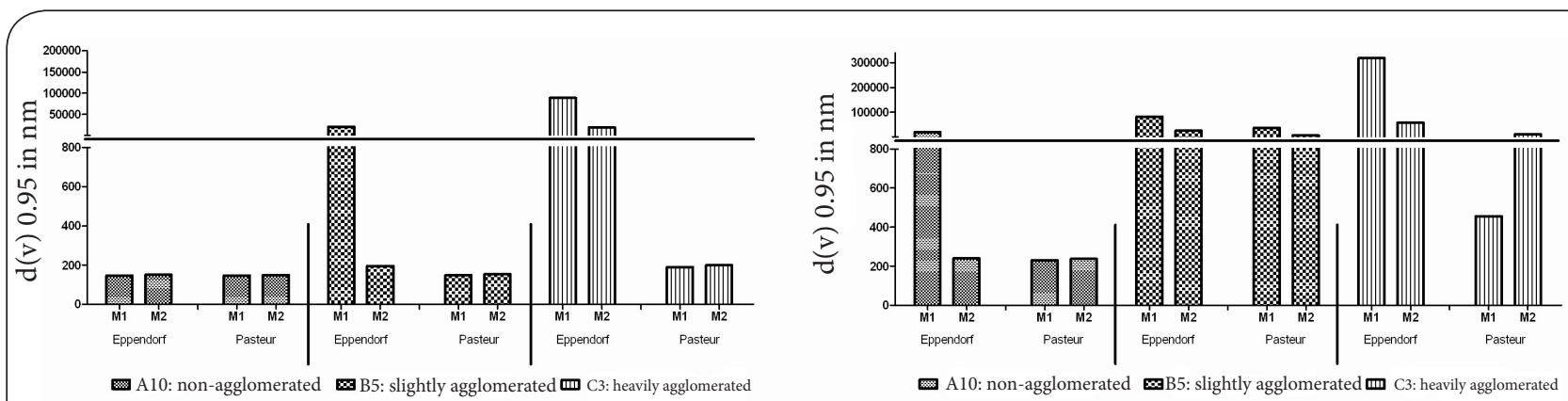

Figure 7. Particle size analysis by laser diffractometry.

Plotted are the d(v)0.50 (left), and the d(v)0.95 (right). Samples were added to the instrument (Mastersizer) by different sampling devices (Eppendorf pipette or Pasteur pipette, respectively) and analyzed using different stirring forces: M1 - 1085 rpm - no sonication; M2 - $2975 \mathrm{rpm}$ - no sonication.

and thus the degree of instability of the formulation. The effect was also observed for the strongly agglomerated sample (C3), however less pronounced. For sample C3 it was also observed, that, when using the Pasteur pipette as sampling instrument, the $\mathrm{d}(\mathrm{v}) 0.95$ and to a minor degree also the $d(v) 0.50$, was smaller, when M2 (higher stirring rate) was applied. The result nicely shows, that large and heavy particles (the addition of the Pasteur pipette does not influence the size) sediment during the measurement if the stirring rate is too low. The larger particles escape from the measuring cell localizing in the tubes of the sample, leading to a smaller size result.

As a consequence of this part of the study it can be stated that also the use of different types of sampling devices can be a pitfall for the size analysis of particles, because different types of sampling instruments can lead to different size results. However again it also opens the opportunity to discriminate samples from each other. Sampling instruments which possess some shear forces, eg. the Eppendorf pipettes used in this study, tend to promote agglomeration of instable samples; again giving the possibility to detect such differences at a very early state of the formulation development.

As another consequence of this study it should by concl- uded it is best to use a Pasteur pipette for the addition of the sample. However, as seen from previous studies $[17,18]$, the sample amount of the sample can also influence the size result (eg. amount of larger particles within the sample). Eppendorf pipettes are much more accurate than Pasteur pipettes. Therefore, a Pasteur pipette should only be used if the results surely are not influenced by the sample amount added. If the size results are known to be concentration dependent or if this effect is not known or investigated, an Eppendorf pipette should be used to ensure reproducible sizing results.

\section{Influence of stirring and ultrasound Analysis by light microscopy and dynamic light scattering \\ From the second part of the study it was shown that the} stirring rate of the instrument can have a very pronounced influence on the size result obtained. Therefore, this effect was investigated in this part of the study in more detail. For this a broad variety of different samples, varying in composition, DLS and microscopic appearance was analyzed (cf. Tables 1 and 2). Prior to size analysis by laser diffractometry samples were analysed using light microscopy and DLS. Based on analysis by light microscopy, 


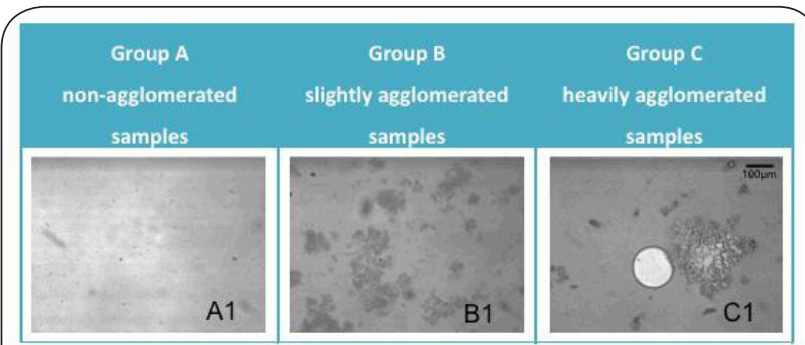

Figure 8. Examples of microscopic images (magnification 160x) of the samples investigated. Left: samples of group A do not contain agglomerates; middle: samples of group B contain agglomerates, which appear loose; right: samples of group $\mathbf{C}$ contain strongly agglomerated particles.

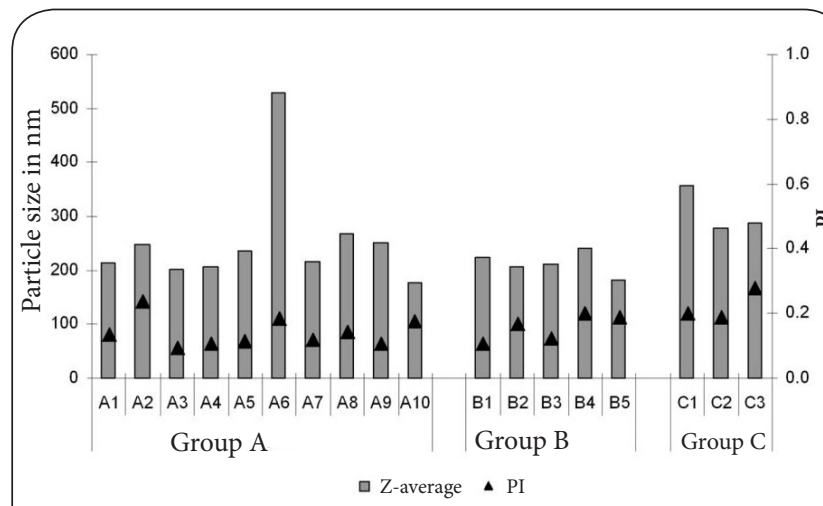

Figure 9. Particle size (z-average) and polydispersity index analyzed by DLS.

No significant difference in size and size distribution are between the different groups. Exception was the sample A6, which possessed relatively higher particle size than other samples, but no agglomeration regarding light microscopy investigations.

samples were grouped into being either non-agglomerated and without detectable $\mu \mathrm{m}$ particles (A samples, group A) or being without $\mu \mathrm{m}$ particles but with some slight/loose agglomerates (B samples, group B). Samples containing agglomerates and/or larger particles were selected to belong to group C (C samples). (Figure 8) shows an example of the microscopic appearance as a representative for each group. (Figure 9) shows the DLS size results of all samples investigated. All particles were in the range between 200 $\mathrm{nm}$ and $350 \mathrm{~nm}$, all polydispersity indices were below 0.3 , i.e., an acceptable broadness in their size distribution. Thus, no large differences between the particles are seen from this sizing technique. There was one exception. Sample A6 (group A) possessed a size of about $530 \mathrm{~nm}$, which is relatively large for this type of nanocarriers. However, light microscopy revealed no larger particles und therefore it was grouped into group $A$.

\section{Size analysis by laser diffractometry}

Size analysis was performed as described in section 3.2.2.
M1 corresponds to a measurement with a stirring speed of 1085 rpm, M2 corresponds to a measurement with 2975 rpm. Furthermore in this part of the study, the possible influence of the ultrasound was investigated. Therefore, in addition to $M 1$ and $M 2$, samples were also analyzed with a third measurement method (M3) which corresponded to $2975 \mathrm{rpm}$ stirring rate and additional sonication throughout the entire measurement. The results of these measurements are shown in Figure 10. Figure 10-left shows the $d(v) 0.50$ values and Figure 10-right shows the $\mathrm{d}(\mathrm{v}) 0.95$ values.

In group A for most of the samples no significant influence of the stirring speed and/or sonication was observed (samples A1-A6). The second part of group A showed an increase in size with increasing stirring speed (samples A7-A9). One exception was sample A10 which showed a decrease in size with increasing stirring rate. The effect observed for sample A10 is due to the use of the Eppendorf pipette and was already seen and discussed in section 3.2.2.

The different effects for the samples A1-A6 and for the samples A7-A9 can be explained as follows: Samples containing no agglomerates or larger particles when analyzed by light microcopy and which did not show any influence of the stirring speed or of sonication on the particle size can be regarded to be very stable (A1-A6). If an increase in the stirring rate or the use of ultrasound leads to an increase in the size of such group A particles, samples aggregate upon the energy input (A7-A9). The effect is due to an in-appropriate stabilizer, which cannot prevent the agglomeration of the particles upon the energy input (cf. Section 3.2.2). The increase in size is due to the energy input into such systems and can therefore be compared to a standard "stress test", being typically performed for eg. emulsions (i.e., stability testing of cosmetic formulations). Thus, this short "stress" test by simply varying the stirring speed might be used as early indicator for the physilal stability of nanodisperse systems upon energy input.

All group B samples and sample C3 decreased in size when the stirring speed was increased. This indicates de-aggregation of the particles due to the energy input. No or little influence was found for the samples C1 and C2 (heavily agglomerated samples). Therefore, changes in size upon changes in the stirring rate can also be used to discriminate loose, slightly agglomerated or heavily agglomerated samples from each other.

Of course agglomeration/de-agglomeration is a size dependent process. Therefore, in theory it was assumed that by analyzing the size over a longer measuring time (i.e., repeated measurements) it should be possible to monitor changes in size over time. In case these changes would vary for the different samples analyzed, again it would be possible to discriminate samples regarding their degree of agglomeration. For example, if larger particles disappear quickly, this means agglomerates within the samples are destroyed quickly upon stirring, indicating 

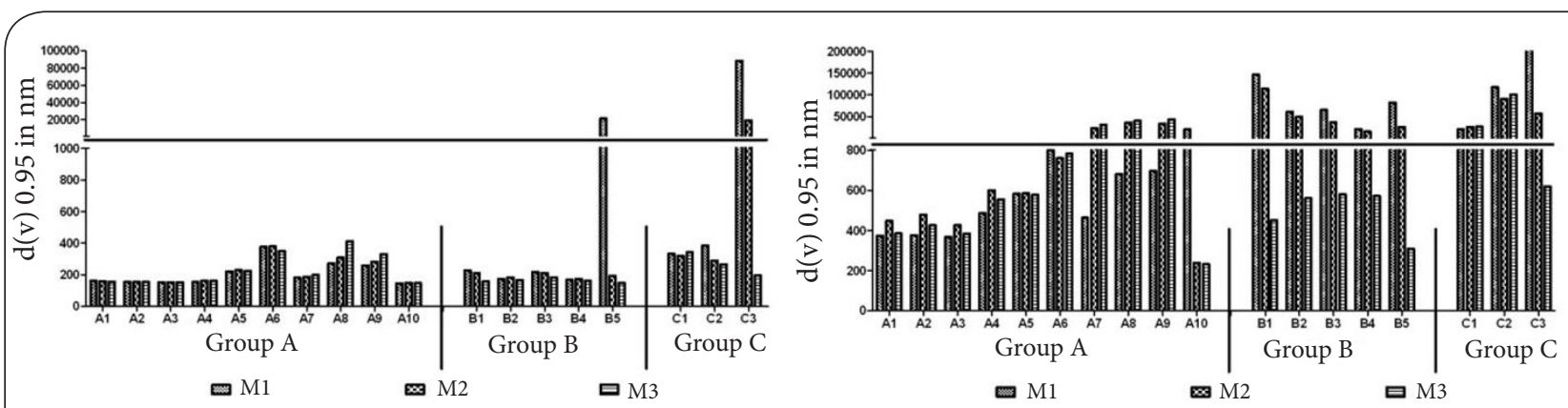

Figure 10. Particle size analysis by laser diffractometry.

Plotted are the d(v)0.50 (left), and the d(v)0.95 (right). Samples were added to the instrument (Mastersizer) with an Eppendorf pipette and analyzed using different measuring conditions, i.e., stirring forces: M1 - $1085 \mathrm{rpm}$ - no sonication; M2 - $2975 \mathrm{rpm}-$ no sonication; M3 - $2975 \mathrm{rpm}$ with sonication.
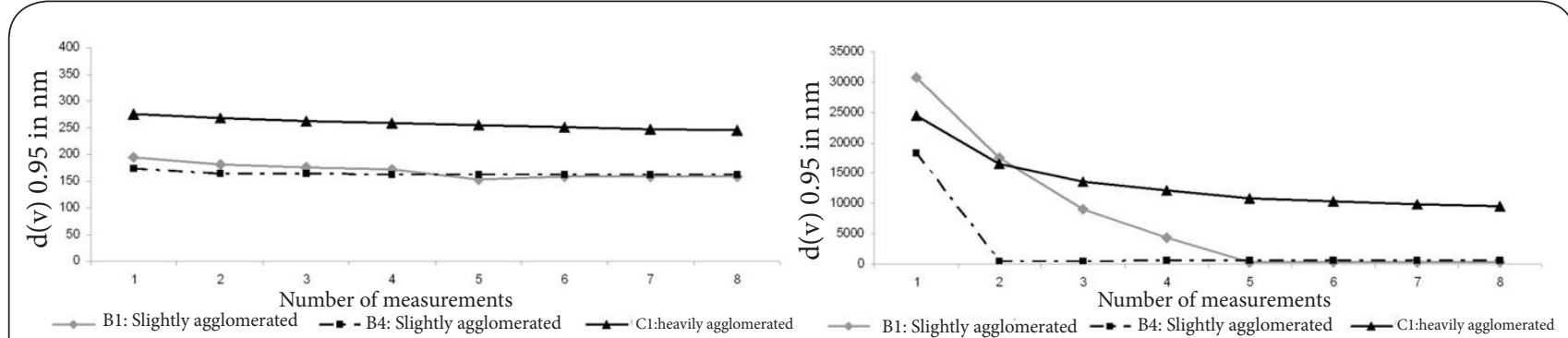

Figure 11. Particle size analysis by laser diffractometry of samples B1, B4 (group B) and sample C1 (group C).

Plotted are the d(v)0.50 (left), and the d(v)0.95 (right). Samples were added to the Instrument with an Eppendorf pipette and analyzed using $2975 \mathrm{rpm}$ as stirring rate with additional sonication (M3, cf. Figure 7).

a very loose agglomeration. A slower decrease in size over time indicates a more pronounced agglomeration and hence a less efficiently stabilized system. If the larger particles remain, the sample is strongly aggregated. Hence, the measurement method would allow for a very simple and fast further discrimination between more or less instable samples. When using measuring media, eg. simulated gastric or intestinal fluid instead of water, it would even be possible to discriminate for more or less stable formulations upon oral administration. Consequently, by analyzing samples this way during formulation development, an earlier discrimination between suitable and non-suitable formulations would be possible, leading to a faster and more successful formulation development by saving time and costs.

Therefore, to investigate the possibility to further discriminate samples regarding the tightness of their agglomerates, samples were analyzed over a longer time (i.e., eight subsequent measurements were performed, instead of four). The results obtained for the samples B1, B4 and C1 are shown in (Figure 11).

Only little changes over time were detect for the $\mathrm{d}(\mathrm{v}) 0.50$ values. However, very clear decreases in size over the time were detected for the $d(v) 0.95$ values. All values decreased exponentially and reached a minimal size after a certain time. However, the decay in size and/or the final size reached were different for each sample. For example, the decay in size was faster for sample B4, when compared to sample B1 and after five measurements similar sizes were obtained for both samples. When comparing the decays in size obtained for sample $\mathrm{B} 1$ and sample $\mathrm{C} 1$, the time required to obtain the minimum size was similar, but the final size reached was much higher for sample $\mathrm{C} 1$, than for sample B1. These results indicate that the agglomeration in sample $B 1$ was more pronounced than for sample B4, i.e., sample B1 is less stable than sample B4, but if enough agitation forces are applied to this sample, the samples will possess the same size (and size related properties). Therefore, the degree of agglomeration is identical. In contrast to this sample C1 contains larger particles than sample B1 which cannot be de-aggregated by agitation, thus the properties of these samples are not identical.

As a result of this study it was therefore found that the stirring rate and/or sonication can strongly influence the size result obtained. Of course this can be a pitfall if not considered and if the measuring conditions are not kept constant between the measurements. Nevertheless, by knowing the possible influence of the stirring speed and by systematically changing it during the size analysis or by monitoring the changes in size over time, manifold 
Kubart et al. Journal of Pharmaceutical Technology \& Drug Research 2013, http://www.hoajonline.com/journals/pdf/2050-120X-2-17.pdf

Table 3. Summary of the pitfalls and opportunities, which are related to the size analysis of submicron particles [16,17,22].

\begin{tabular}{|c|c|c|c|c|}
\hline $\begin{array}{l}\text { parts of the performance } \\
\text { of size analysis }\end{array}$ & $\begin{array}{l}\text { parameters which } \\
\text { might influence } \\
\text { the size result }\end{array}$ & pitfalls & opportunities & references \\
\hline \multirow{3}{*}{ prior to the measurement } & $\begin{array}{l}\text { selection of } \\
\text { measuring medium }\end{array}$ & $\begin{array}{l}\text { Measuring in a medium, which is able to } \\
\text { dissolve the particles, can lead to incorrect } \\
\text { results due to the dissolution of the particles. }\end{array}$ & $\begin{array}{l}\text { A fast dissolution test could be performed using } \\
\text { a suitable dissolution medium as measuring } \\
\text { medium. }\end{array}$ & {$[17,22]$} \\
\hline & $\begin{array}{l}\text { preparation of } \\
\text { sample for the } \\
\text { measurement }\end{array}$ & $\begin{array}{l}\text { Larger particles, i.e., agglomerates might be } \\
\text { destroyed during the shaking prior to sampling. }\end{array}$ & $\begin{array}{l}\text { A possible instability of a sample could be detected } \\
\text { early by analysis of samples collected from } \\
\text { different positions of non-shaken samples. }\end{array}$ & cf. Section 3.1 \\
\hline & $\begin{array}{l}\text { selection of the } \\
\text { sampling device }\end{array}$ & $\begin{array}{l}\text { Depending on sampling device, particles might } \\
\text { lead to agglomeration or to de-agglomeration of } \\
\text { particles and consequently incorrect results. }\end{array}$ & $\begin{array}{l}\text { A possible instability of a sample could be detected } \\
\text { early, eg. by using a sampling instrument which } \\
\text { possesses some shear forces and consequently } \\
\text { promotes agglomeration of instable samples. }\end{array}$ & cf. Section 3.2 \\
\hline \multirow{3}{*}{ measurement } & sampling & $\begin{array}{l}\text { Sampling from different positions of non- } \\
\text { shaken samples can lead to different size results } \\
\text { and thus to non-reproducible measurements. }\end{array}$ & $\begin{array}{l}\text { A possible instability of a sample could be detected } \\
\text { early by analysis of samples collected from } \\
\text { different positions of non-shaken samples. }\end{array}$ & cf. Section 3.1 \\
\hline & $\begin{array}{l}\text { stirring speed/ ultra } \\
\text { sound }\end{array}$ & $\begin{array}{l}\text { Depending on stirring speed and application of } \\
\text { sonication particles might lead to agglomera- } \\
\text { tions or de-agglomerations of particles. }\end{array}$ & $\begin{array}{l}\text { A discrimination between more and less instable } \\
\text { samples and the determination of the tightness } \\
\text { of agglomerates is possible by applying different } \\
\text { stirring speeds and/or sonication. }\end{array}$ & cf. Section 3.3 \\
\hline & $\begin{array}{l}\text { additional } \\
\text { technique }\end{array}$ & $\begin{array}{l}\text { With using the additional technique the detec- } \\
\text { tion of larger particles beside small sized main } \\
\text { population could failure. }\end{array}$ & $\begin{array}{l}\text { Using the additional technique extents the } \\
\text { measuring range. Thus, the measuring of } \\
\text { submicron particles is possible. }\end{array}$ & [16] \\
\hline analysis of results & optical parameters & $\begin{array}{l}\text { Using incorrect optical parameters can lead to } \\
\text { incorrect particle size and distribution results. }\end{array}$ & - & {$[16,22]$} \\
\hline
\end{tabular}

useful information of the samples can be gained. Again this gives the opportunity to turn a possible pitfall into a great opportunity to gain more insight into sample stability.

\section{Summary}

In previous studies it was shown that optical parameters, additional techniques and the dissolution of particles during the measurement have a very pronounced effect on the particle size obtained. In this study further parameters that can influence the size results obtained were studied. The results of all these studies, i.e., the parameters which were identified to possibly influence the size results, as well as the pitfalls and the opportunities, which are related to the size analysis of submicron particles, are summarized in (Table 3). If all these parameters are considered, laser diffractometry can be used as a powerful technique during the development of new formulations and for the characterization (eg. quality control) of submicron particles in general.

\section{Conclusion}

The results of the study revealed that sampling position, sampling device and stirring speed as well as the use of ultrasound can have a tremendous influence on the size effect obtained. As a consequence, to ensure meaningful size results, all these parameters must be established for each type of sample and must be kept constant during size analysis by laser diffractometry. As a second consequence, similar to the optical parameters and other measuring or analysis conditions [16-18], for a possible comparison of the results, all these parameters used must be mentioned, when publishing size data obtained by laser diffractometry. Size data without these specifications are meaningless. Besides these pitfalls, the systematic use of different measuring conditions can be used for improved formulation development, as it enables the discrimination between "good and bad formulations" at a very early stage of the development, maybe even replacing some longterm storage tests. In conclusion, laser diffractometry is an important sizing method for the characterization of nanosized systems. It involves many pitfalls which can be overcome and even exploited as advantages if understood.

\section{Competing interests}

The authors declare that they have no competing interests.

Authors' contributions

Senem Acar Kübart carried out the particle size measurements and light microscopy investigations of part 2 and 3 of the study, and helped to draft the manuscript. Cornelia M Keck carried out the particle size measurements and light microscopy investigations of part 1 of the study, and drafted the manuscript. All authors read and approved the final manuscript.

Acknowledgement and funding

The authors would like to thank the company PharmaSol GbmH, Berlin for scientific support and the author Senem Acar Kübart would like to thank the Deutscher Akademischer Austauschdienst - DAAD (Kennziffer No. $A / 08 / 76475)$ for their financial support.

Publication history

Received: 20-Feb-2013 Revised: 19-Apr-2013

Accepted: 22-Apr-2013 Published: 27-Apr-2013

\section{References}

1. Sarker DK: Engineering of nanoemulsions for drug delivery. Curr Drug Deliv 2005, 2:297-310. | Article | PubMed

2. McClements DJ and Rao J: Food-grade nanoemulsions: 
formulation, fabrication, properties, performance, biological fate, and potential toxicity. Crit Rev Food Sci Nutr 2011, 51:285-330. | Article | PubMed

3. Rajpoot $P$, Pathak $K$ and Bali V: Therapeutic applications of nanoemulsion based drug delivery systems: a review of patents in last two decades. Recent Pat Drug Deliv Formul 2011, 5:163-72. | Article | PubMed

4. Crommelin DJ and Storm G: Liposomes: from the bench to the bed. J Liposome Res 2003, 13:33-6. | Article | PubMed

5. Storm G, Wilms HP and Crommelin DJ: Liposomes and biotherapeutics. Biotherapy 1991, 3:25-42. | Article | PubMed

6. Kreuter J: Nanoparticles and microparticles for drug and vaccine delivery. J Anat 1996, 189 ( Pt 3):503-5. | PubMed Abstract | PubMed Full Text

7. Tosi G, Costantino L, Ruozi B, Forni F and Vandelli MA: Polymeric nanoparticles for the drug delivery to the central nervous system. Expert Opin Drug Deliv 2008, 5:155-74. | Article I PubMed

8. Hasadsri L, Kreuter J, Hattori H, Iwasaki T and George JM: Functional protein delivery into neurons using polymeric nanoparticles. J Biol Chem 2009, 284:6972-81. | Article | PubMed Abstract | PubMed Full Text

9. Muller RH, Gohla S and Keck CM: State of the art of nanocrystals--special features, production, nanotoxicology aspects and intracellular delivery. Eur J Pharm Biopharm 2011, 78:1-9. | Article | PubMed

10. Muller RH, Shegokar R and Keck CM: 20 years of lipid nanoparticles (SLN and NLC): present state of development and industrial applications. Curr Drug Discov Technol 2011, 8:207-27. | Article | PubMed

11. Müller RH, Shegokar R, Gohla S and Keck CM: Nanocrystals: Production, Cellular Drug Delivery, Current and Future Products. In Prokop A (Ed.), Intracellular Delivery: Fundamentals and Applications, Fundamental Biomedical Technologies 2011, 411-432. | Book

12. Meunier F, Prentice HG and Ringden O: Liposomal amphotericin B (AmBisome): safety data from a phase II/III clinical trial. J Antimicrob Chemother 1991, 28 Suppl B:83-91. | Article | PubMed

13. Malvern: Handbook Zetasizer Nano ZS, Malvern Instruments, Worcestershire, UK. | Website

14. Allen T: Ceramic Powder Science, Advances in Ceramics. 1987, 721. | Website

15. Wingfield M: Partikelgrösse und Partikelform (morphologische Parameter) mit modernen Methoden bestimmen: Methodenvergleich - Fehlerbetrachtung Variabilität. 2009. | Pdf

16. Keck CM and Muller RH: Size analysis of submicron particles by laser diffractometry-- $90 \%$ of the published measurements are false. Int J Pharm 2008, 355:150-63. | Article | PubMed

17. Keck CM: Particle size analysis of nanocrystals: improved analysis method. Int J Pharm 2010, 390:3-12. | Article | PubMed

18. Keck CM: Cyclosporine Nanosuspensions: Optimised Size Characterisation \& Oral Formulations. 2006. I Pdf

19. Leuenberger H: Martin Pysikalische Pharmazie, Wissenschaftliche Verlagsgesellschaft, Stuttgart, 4th Ed. 2002. p. 587-589.

20. Lagaly G, Schulz O and Zimehl R: Dispersionen und
Emulsionen. 1999. | Book

21. Müller RH: Colloidal Carriers for Controlled Drug Delivery and Targeting. 1991. | Book

22. Method validation for laser diffraction measurements (MRK671-01), Malvern Instruments, Worcestershire, Malvern, Mastersizer 2000 Application Note, UK. I Pdf

\section{Citation:}

Kübart SA and Keck CM: Laser diffractometry of nanoparticles: frequent pitfalls \& overlooked opportunities. journal of Pharmaceutical Technology and Drug Research 2013, 2:17.

http://dx.doi.org/10.7243/2050-120X-2-17 